FERMILAB-Pub-93/086-T

CERN-TH-6868/93

$\mathrm{DTP} / 93 / 24$

hep-ph/9305220

\title{
Single Lepton Production at Large Transverse Momentum
}

\author{
W. T. Giele \\ Fermi National Accelerator Laboratory, P. O. Box 500, \\ Batavia, IL 60510, U.S.A. \\ E. W. N. Glover \\ Physics Department, University of Durham, \\ Durham DH1 3LE, England \\ and \\ David A. Kosower' \\ Theory Division, CERN, \\ CH-1211 Geneva 23, Switzerland \\ kosower@nxth02. cern.ch
}

April 1993

\begin{abstract}
We study the production of single isolated leptons at large transverse momentum, $p_{T}^{\ell}>M_{W} / 2$. The dominant source of such leptons is production of an on-shell $W$ boson recoiling against a hard jet. Vetoing this jet forces the $W$ boson to be produced off resonance and significantly reduces the standard model cross section, thereby enhancing the discovery prospects for non standard model physics. A significant number of events have the topologically interesting signature of a large $p_{T}$ lepton balancing a jet with little measured missing transverse energy.
\end{abstract}

\footnotetext{
${ }^{1}$ On leave from the Centre d'Etudes of Saclay, F-91191 Gif-sur-Yvette cedex, France
} 
Just over a decade ago, the $W$ and $Z$ bosons were discovered at the CERN Spp $\overline{\mathrm{S}}$ by observing isolated leptons at large transverse momentum. In the case of the $W$ boson, the lepton was accompanied by significant missing transverse momentum due to the neutrino escaping the detector. The cross section for these processes is roughly a nanobarn. In the near future, experiments at the Fermilab Tevatron expect to collect an integrated luminosity of $100 \mathrm{pb}^{-1}$ and are thus sensitive to cross sections of the order of $0.1 \mathrm{pb}$. At such cross sections, one might hope to find hints of physics beyond the standard model.

Let us consider, for example, events with isolated leptons and jets, where the lepton has a large transverse momentum, $p_{T}^{\ell}>70 \mathrm{GeV}$, or events with leptons and missing transverse energy where the transverse mass, $M_{T}(\ell, \nu)>140 \mathrm{GeV}$. These events are topologically interesting and could represent the signal of new physics. One example is a $W^{\prime}$ boson of extended gauge models [1]. However, one must first understand the possible sources of such events in standard model processes, and that is our purpose in this Letter.

We begin by considering the lowest order process,

$$
q \bar{q} \rightarrow W^{ \pm} \rightarrow \ell^{ \pm} \nu
$$

When $p_{T}^{\ell} \sim M_{W} / 2$, the $W$ boson can be produced on-resonance. However, if we demand that the lepton transverse momentum be much larger, the $W$ boson will be forced off its resonance and the cross section will fall sharply. This is shown in Fig. 1 where we plot the total cross section for the production of a single species of charged lepton with $p_{T}^{\ell}>p_{T}^{\text {cut }}$ and rapidity $\left|\eta^{\ell}\right|<2$ in association with a missing transverse energy $E_{T}^{\text {miss }}>20 \mathrm{GeV}$. Motivated by the recent though preliminary $\mathrm{H} 1$ data on deep inelastic scattering [2] we have restricted our attention to the MRSD-' [3] structure functions. However, since we are dealing with a process at relatively large $x, x \sim 0.05$, different choices for the input structure functions do not make a significant difference. We observe that for $p_{T}^{\text {cut }}=70 \mathrm{GeV}$, the cross section is still about $1 \mathrm{pb}$.

At next-to-leading order, one must consider radiative processes such as,

$$
q \bar{q} \rightarrow W^{ \pm} g \rightarrow \ell^{ \pm} \nu g, \quad q g \rightarrow W^{ \pm} q \rightarrow \ell^{ \pm} \nu q
$$

which can give rise to a jet. In this case, the definition of $E_{T}^{\text {miss }}$ is slightly modified to be the transverse imbalance of all energy deposited in a calorimeter which extends to $|\eta|=4$. Using the results of ref. [4], we show the next-to-leading order corrections to the total cross section as a function of $p_{T}^{\text {cut }}$ in Fig. 1. These next-to-leading corrections alter the predicted rate for the inclusive process given a large $p_{T}^{\text {cut }}$, by a large factor - a factor of nearly five at $p_{T}^{\text {cut }}=70 \mathrm{GeV}$. The reason for this increase is straightforward; requiring a large lepton transverse momentum no longer forces the $W$ far off resonance, since it can acquire a large transverse momentum by recoiling against a jet. This additional transverse momentum is then inherited by the daughter lepton. Thus, there is a trade off between a larger subprocess center of mass energy combined with a factor of $\alpha_{s}$ and the off-resonance suppression. To show that this is indeed the case, we also show the cross section for the production of large 


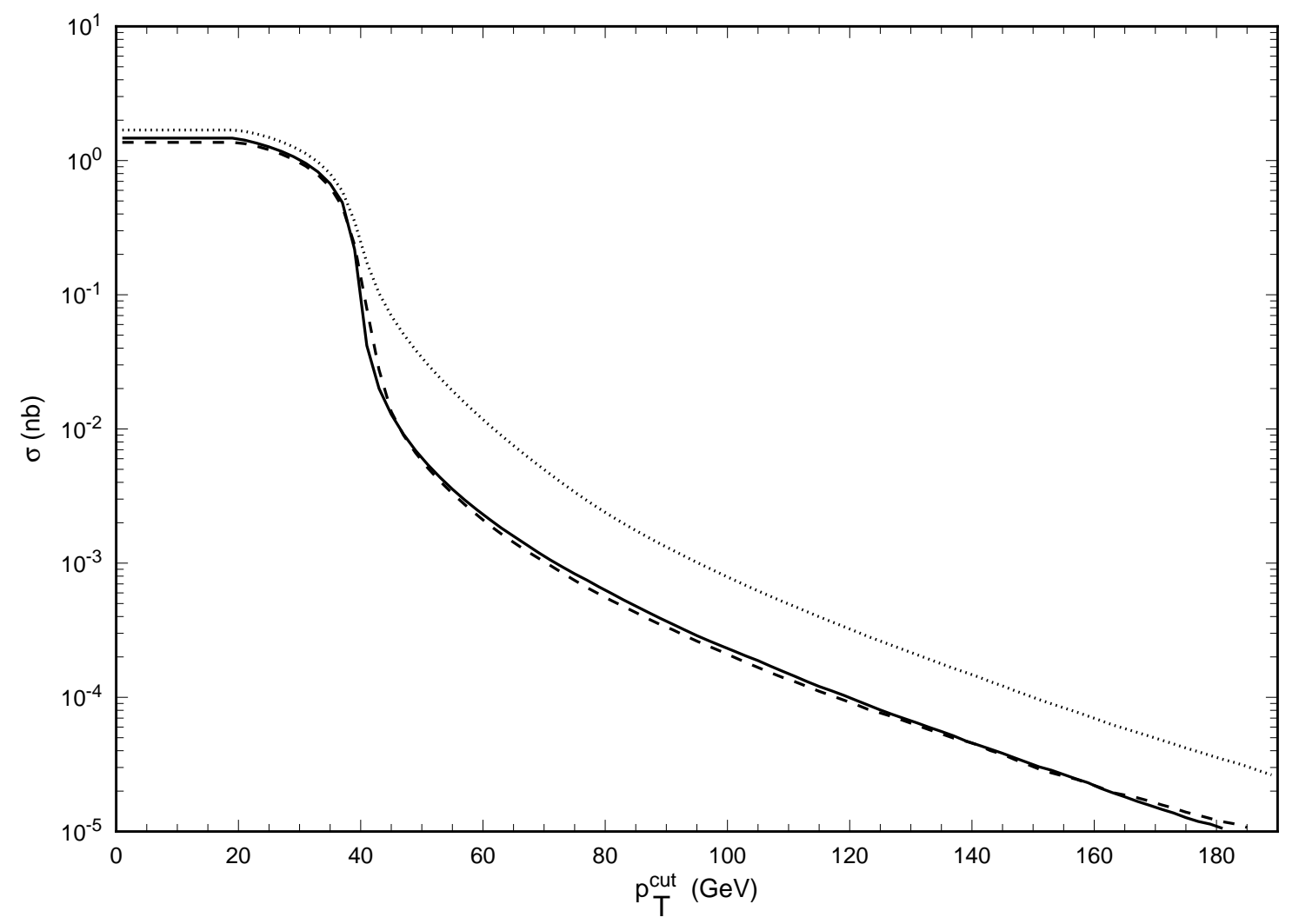

Figure 1: The total cross section for events containing leptons with transverse momentum $p_{T}^{\ell}>p_{T}^{\text {cut }}$ in association with missing transverse energy. Both leading order (solid line) and next-to-leading order (dotted line) predictions are shown. We also show the next-to-leading order prediction for the jet-veto rate where no hadronic cluster with $E_{T}>10 \mathrm{GeV}$ and $|\eta|<4$ is observed in the event (dashed line).

transverse momentum leptons at next-to-leading order where we veto events by requiring that no hadronic cluster with transverse energy $E_{T}>10 \mathrm{GeV}$ and $|\eta|<4$ be observed. As expected, the genuine $\mathcal{O}\left(\alpha_{s}\right)$ corrections to the 0 -jet rate are small. This gives us confidence in the convergence of the perturbation series for this quantity. We see that the majority of events containing a large transverse momentum electron fail this veto.

It is also instructive to examine the transverse mass distribution for these events. The transverse mass $M_{T}$ is defined as

$$
M_{T}=\sqrt{2 p_{T}^{\ell} E_{T}^{\text {miss }}(1-\cos \phi)}
$$

where $\phi$ is the azimuthal angle between the lepton momentum and the missing transverse energy vector. In Fig. 2, we show the next-to-leading order predictions for this distribution for both the inclusive reaction and reactions with a jet veto (using the same cuts as in the previous paragraph) and $p_{T}^{\ell}>p_{T}^{\text {cut }}=70 \mathrm{GeV}$. The peak at $M_{T} \sim M_{W}$ arises from events where the large lepton $p_{T}$ is balanced by a hard jet. The second peak is perhaps unexpected, 


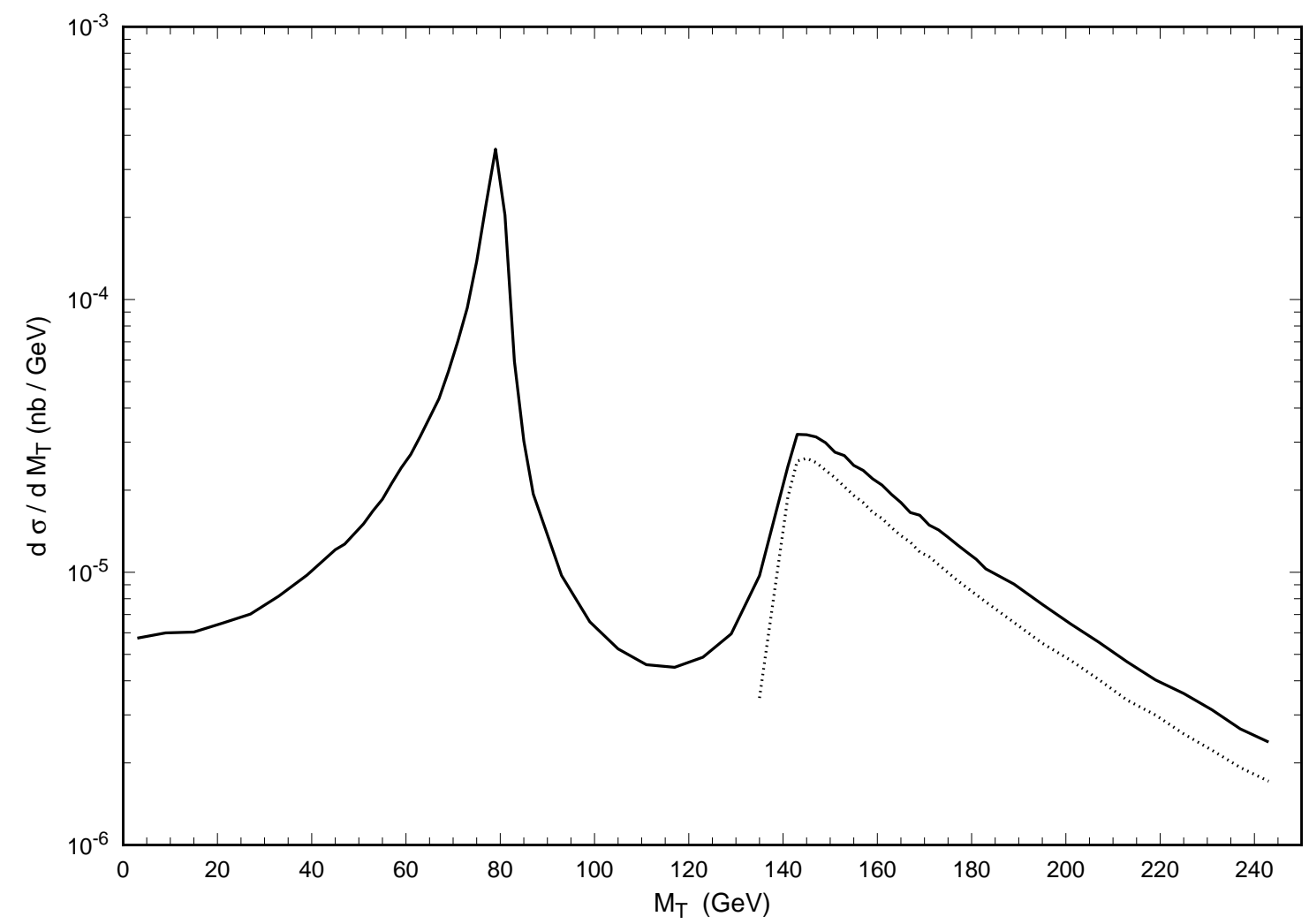

Figure 2: The inclusive next-to-leading order transverse mass distribution for events containing a charged lepton with $p_{T}^{\ell}>70 \mathrm{GeV},\left|\eta^{\ell}\right|<2$ and $E_{T}^{\text {miss }}>20 \mathrm{GeV}$. The rate for events passing a jet veto is shown dotted.

however. Applying a jet veto eliminates the first peak but not the second, indicating that the second peak arises when the transverse momentum of the lepton and neutrino approximately balance and there is little hadronic energy in the event. The $W$ boson is far off shell and this contribution is restricted to the region $M_{T} \gtrsim 2 p_{T}^{\text {cut }}=140 \mathrm{GeV}$. The total cross section in the region $M_{T}>140 \mathrm{GeV}$ is about $1 \mathrm{pb}$ which should be observable using the current data sample. But one must exercise caution in isolating events in this region: the inclusive spectrum might suggest that the second peak is a signature of new physics in spite of its artifactual origin in the cuts.

One may also consider, as mentioned in the introduction, the class of events where the charged lepton recoils against a jet, yet the missing transverse energy is small. In Fig. 3, we show the leading and next-to-leading order predictions for the missing transverse energy distribution in events where one jet? with transverse momentum $E_{T}^{\text {jet }}>25 \mathrm{GeV}$, pseudorapidity $\left|\eta^{\text {jet }}\right|<3$ and cone size $\Delta R=0.7$ is observed in association with a charged

\footnotetext{
${ }^{2}$ The jet axis and energy are obtained by adding the four-momenta of partons contained in a cone of size $\Delta R$.
} 


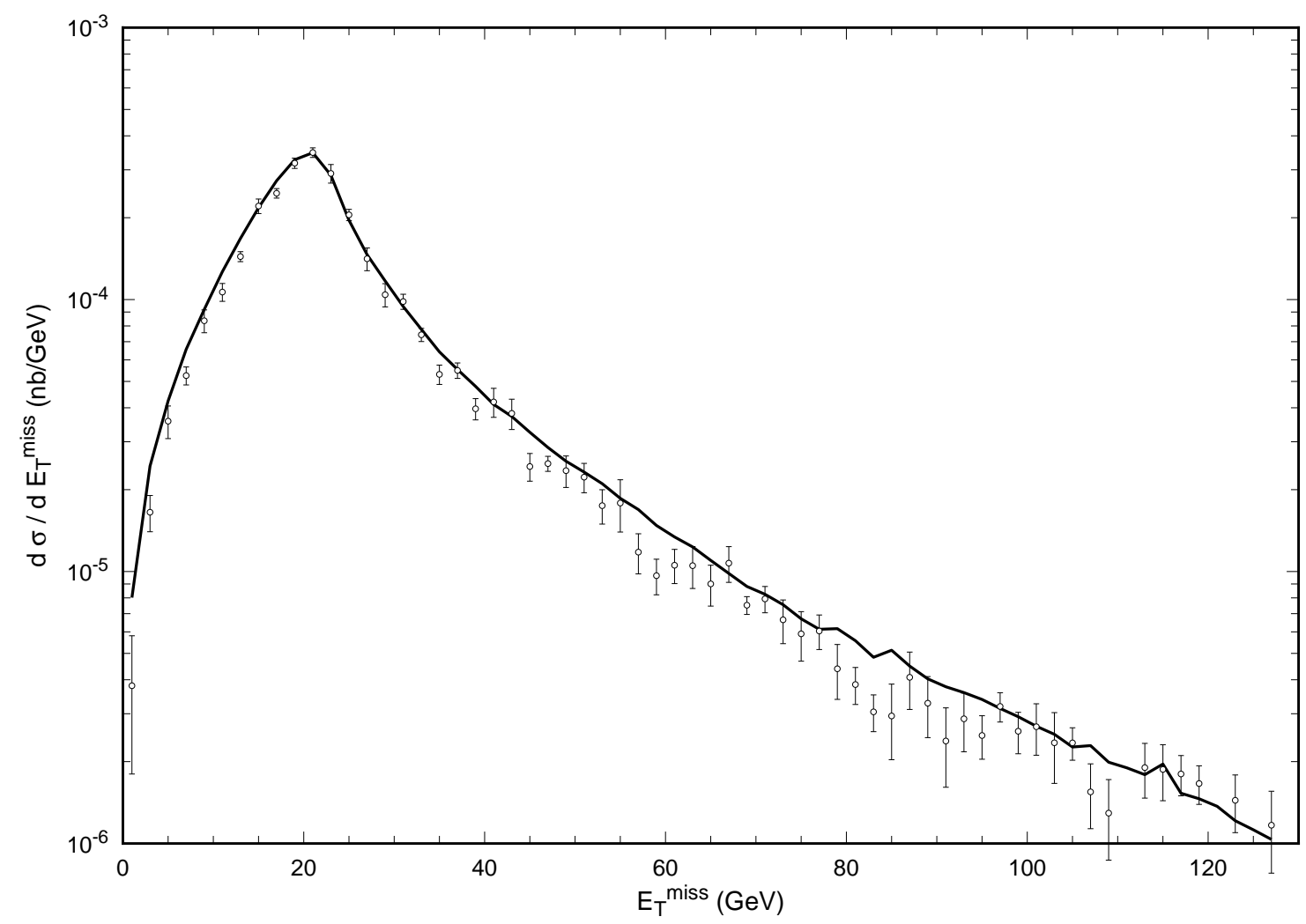

Figure 3: The leading order $E_{T}^{\text {miss }}$ distribution for events containing a charged lepton with $p_{T}^{\ell}>70 \mathrm{GeV},\left|\eta^{\ell}\right|<2$ and a single jet $E_{T}^{\text {jet }}>25 \mathrm{GeV}$. The next-to-leading order rate is shown as points.

lepton possessing $p_{T}^{\ell}>p_{T}^{\text {cut }}=70 \mathrm{GeV}$. The next-to-leading order QCD corrections do not alter the shape of the distribution. The largest cross section occurs when the $W$ boson is on shell. In this case, for back-to-back lepton and neutrino, we expect

$$
E_{T}^{\text {miss }} \sim \frac{M_{W}^{2}}{4 p_{T}^{\text {cut }}} \sim 23 \mathrm{GeV},
$$

and a peak near this value can be seen. Although most events do contain noticeable missing tranverse energy, the total cross section for lepton + jet events with less than $20 \mathrm{GeV}$ of missing energy is appreciable (about $2 \mathrm{pb}$ ).

In this letter we have examined two classes of events with high transverse momentum leptons, isolated lepton and lepton + jet events. One must take care, when imposing a high transverse momentum cut on the lepton, to use next-to-leading order calculations in order to verify that deviations from predictions are indeed a signal of new physics. We note that the standard model contains other possible backgrounds such as $\tau^{+} \tau^{-}$and heavy flavour production which we have not estimated. 
The first class contains no jet, a possible signal for an object such as a $W^{\prime}$ boson or, alternatively, for deviations from the standard model due to other sources of higher-dimension operators. In these events, it is essential to impose a jet veto. Such a veto will not reduce the $W^{\prime}$ boson signal but will reduce the standard model background by a large factor, perhaps as large as 5. One can simply count the number of lepton events and compare them with the predictions of Fig. 1. This cross section is largely unaffected by next-to-leading order corrections; the data with a low lepton transverse momentum cut imposed can be used to normalize the theoretical prediction to the data.

The second class of events contains a jet in addition to a lepton. Some exhibit the striking feature of a high momentum jet balancing a lepton, while the missing transverse energy is

small. With a luminosity as small as $25 \mathrm{pb}^{-1}$ we can expect of the order of 50 events with the following characteristics: $p_{T}^{\ell}>70 \mathrm{GeV}$ and $E_{T}^{\text {miss }}<20 \mathrm{GeV}$ balanced by a large $E_{T}$ jet.

We thank the Fermilab ACPMAPS project for providing computer time for some of the calculations described above. In addition, E.W.N.G. and D.A.K. wish to thank the Fermilab theory group for its hospitality during the completion of the work described above.

\section{References}

[1] See for example, J. Pati and A. Salam, Phys. Rev. D10, 275 (1974); R.E. Marshak and R.N. Mohapatra, Phys. Let. B91, 222 (1980).

[2] A. de Roeck, H1 Collaboration. Preliminary data presented at the HERA workshop in Durham, March 1993.

[3] A.D. Martin, R.G. Roberts and W.J. Stirling, Durham preprint DTP/92/80 (1992).

[4] W.T. Giele, E.W.N. Glover and D.A. Kosower, Fermilab preprint FERMILAB-Pub92/230-T (1992), to be published in Nucl. Phys. B. 
This figure "fig2-1.png" is available in "png" format from: http://arxiv.org/ps/hep-ph/9305220v2 
This figure "fig3-1.png" is available in "png" format from: http://arxiv.org/ps/hep-ph/9305220v2 
This figure "fig4-1.png" is available in "png" format from: http://arxiv.org/ps/hep-ph/9305220v2 